\title{
Quantifying the Dimensions of Achilles Tendon Insertional Area Using Ultrasound Imaging A Validity and Reliability Study
}

\author{
N.H. Alghamdi ${ }^{1}, 2$, M. Killian ${ }^{3}$, B. Aitha ${ }^{4}$, R.T. Pohlig ${ }^{1}$, K. Grävare Silbernagel ${ }^{1}$ \\ 1 Department of Physical Therapy, University of Delaware, Newark, DE, USA \\ 2 Department of Physical Therapy, King Abdulaziz University, Jeddah, Saudi Arabia \\ ${ }^{3}$ Department of Biomedical Engineering, University of Delaware, Newark, DE, USA \\ 4 School of Nursing, University of Delaware, Newark, USA
}

\section{CORRESPONDING AUTHOR:}

Karin Grävare Silbernagel

Department of Physical Therapy

University of Delaware

540 South College Ave

Newark, DE 19713, USA

E-mail: Kgs@udel.edu

DOI:

10.32098/mltj.04.2019.09

LEVEL OF EVIDENCE: 3

\begin{abstract}
SUMMARY
Background. The purpose of this study was to evaluate the reliability and validity of using US imaging for measuring Achilles tendon insertion area to the calcaneus.

Methods. Sixteen cadaveric tendons were used to compare the agreement between Achilles tendon insertion length measurements from US images with actual measurements after dissection. In addition, test-retest reliability was performed on six healthy subjects for tendon insertion length (IL), before calcaneal bone cross-sectional area (CSA) and tendon insertion angle (IA). The reliability and validity of the measurements was analyzed with intra-class correlation coefficient (ICC) and standard error of measurement (SEM).

Results. There were significant differences and poor to good agreement between US measurements and Anthropometric measurements. Using cadaveric tendons images, the measurements agreement between the observers were good to excellent for IL, bone- to-insertion length and width before calcaneal bone ( $\left.\mathrm{ICC}_{24}=.784, .935, .952\right)$. Test-retest reliability was excellent for US measurements for tendon IL $\left(\mathrm{ICC}_{2.4}=.977, \mathrm{SEM}=0.53 \mathrm{~mm}\right.$ ), bone to IL ( $\mathrm{ICC}_{2.4}=.96$, $\mathrm{SEM}=0.44 \mathrm{~mm}$ ), width before calcaneal bone (ICC $\left.{ }_{2,4}=.946, \mathrm{SEM}=0.51 \mathrm{~mm}\right), \mathrm{CSA}\left(\mathrm{ICC}_{2,4}=.918\right.$, $\left.\mathrm{SEM}=5.93 \mathrm{~mm}^{2}\right)$, and $\mathrm{IA}\left(\mathrm{ICC}_{2.4}=.933, \mathrm{SEM}=1.9^{\circ}\right)$ on healthy tendons.

Conclusions. US imaging was found to be reliable and valid to determine IL, width, and CSA of the Achilles tendon. The use of US imaging to determine Achilles tendon insertional size may be beneficial for evaluating the structural changes on disease development and progression.
\end{abstract}

\section{KEY WORDS}

ultrasound imaging; achilles tendon; insertion; enthesitis; tendon footprint

\section{BACKGROUND}

The strongest tendon in the human body is the Achilles tendon; nevertheless, it is often injured in active individuals who participate in running and jumping sports $(1,2)$. Clinical symptoms include a combination of local swelling and pain with impaired performance (2). Achilles tendinopathy can be distinguished into two types based on pain and swelling location, proximal mid-portion $(2-6 \mathrm{~cm}$ proximal to tendon insertion) and distal insertional (insertion to calcaneus). Insertional Achilles tendinopathy accounts for $20 \%$ to $25 \%$ of reported Achilles tendon injuries (3-5). Differential diagnosis between Achilles tendinopathy (mid-portion or insertional) and other Achilles pathology like partial rupture, bursitis, and paratendionpathy can be made by clinical examination and primary imaging modalities such as ultrasound (US) and magnetic resonance imaging (MRI) (6-8). Using US imaging has become popular in both clinical and research settings because it is easy to diagnose superficial musculoskeletal injuries. US can also be used to measure soft tissue structures and follow changes over time due to treatment.

Insertional Achilles tendon pain has been reported to be due to pathologies such as insertional tendinopathy, retrocalcaneal bursitis, superficial calcaneal bursitis, calcification, and boney deformity (2). The variety of affected tissues (bone, bursa, and tendon) might explain the recalcitrant nature 
of the injury and why treating it is considered a challenge. It has been found that $24.6 \%$ of patients with insertional Achilles tendinopathy responded to non-surgical treatment compared to $45.5 \%$ of patients with midportion Achilles tendinopathy (9). Also, the various tissues around the Achilles tendon insertion are subject to different type of stresses during walking, running, and jumping, whereas in the midportion there is a more uniform stress of the tendon. Altered loading at the insertion may damage and injure the tissue and over time lead to structural changes such as formation of calcification and boney deformities at the insertional point $(10,11)$. Haglund's deformity may cause impingement that stresses the soft tissue at the posterior heel. One study used X-ray to measure the change of boney deformity in Haglund's deformity (12). However, soft tissues cannot be evaluated with X-ray. Although insertional Achilles tendinopathy has been evaluated with US imaging, no prior studies have measured and validated the structural geometry at the insertion using US imaging (13). Having a valid and reliable method for measuring Achilles tendon insertion, may be beneficial for understating the disease process and treatment effectiveness for insertional Achilles tendinopathy.

US imaging is a safe, quick, and inexpensive method for diagnosing tendon injuries $(14,15)$. It has become the preferable modality for clinicians' screening and a valuable tool for researchers to compare and describe injured structures $(14,15)$. US imaging has been found to be reliable and valid for evaluating Achilles tendon length, thickness, cross-sectional area, and the calcification length in patient with insertional Achilles tendinopathy (16-19). However, validated and repeatable methods for evaluating the reliability of US imaging to quantify the insertional Achilles tendon size have yet to be established.

The primary purposes of this study were to 1) assess the reliability and validity, and procedural reliability of US imaging for measuring the Achilles tendon insertion on the calcaneus, and 2) determine measurement error and smallest detectable change (SDC) values for these US imaging measurements.

\section{MATERIALS AND METHODS}

Our study consists of two parts, the first was to evaluate the validity of the measurements on US imaging and the second part of this study was to evaluate test-retest procedural reliability and measurement stability for these US imaging measurements. Procedural reliability was defined as the ability of examiners to consistently repeat the entire US imaging process, including imaging acquisition and measurements on the images.

Eight cadavers (16 tendons, age range between 70 to 85 years old, 3 males and 5 females) were used; all cadavers were donated for medical education and research purposes, and prior consent was given by the donor and/or next relatives. The US images from the cadavers were also used to evaluate inter-rater reliability of the measurements from the images. For the test-retest procedural reliability, two assessments separated by 30 minutes were performed on six healthy young subjects (12 tendons), with no recent injury of Achilles tendon (age range 19-30,3 male and 3 female). Approval for this study was obtained from institutional review board at the University of Delaware. All healthy participants received verbal and written information about the study and consent was obtained. The article complies with the ethical and scientific standards of Muscle, Ligaments and Tendons Journal (20).

\section{Imaging of Achilles tendon insertions}

In this study, all US images were obtained by using LOGIQ-e US (GE Healthcare) system, and recorded using a wideband linear array probe $(5.0-12.0 \mathrm{MHz})$ with B-mode at $10 \mathrm{MHz}$. The first author, who has more than 6 months of experience with US imaging of Achilles tendon, and had passed the reliability training of the research lab, collected the US images from both cadavers and healthy subjects.

The position for both cadavers and healthy subjects was prone with the ankle position for healthy subjects at resting position and out of the table edge and for cadaver's ankle position was between $15^{\circ}$ to $65^{\circ}$ of plantarflexion. During image acquisition from the cadavers, the US transducer was covered in a disposable plastic sleeve, and the gain was adjusted for each image to enhance quality. A gel pad was used between the US probe and the heel to improve the visibility of the tendon insertion.

Image collection started by scanning the Achilles tendon from musculotendon junction to the insertion. Then we obtained four long-axis images of the Achilles tendon insertion to measure the insertion length and tendon angle. We then identified the posterior-superior part of the calcaneus. A piece of tape with $1 \mathrm{~mm}$ width was placed on the center of the probe to generate shadow as an indicator on the image (figure 1). The skin was marked with a pen just at the tape site when the shadow was aligned with the posterior-superior part of the calcaneus. At this location, we obtained four short-axis images of the tendon for measuring tendon width and cross-section area. All US images were then transferred to another computer for performing measurements, using OsiriX Lite version 8.0.2 (https://www.osirix-viewer.com/ osirix/osirix-md/download-osirix-lite/).

The skin of healthy participants were cleaned between measurements, and they were allowed to leave the lab and came back after 30 minutes for the second test session. 


\section{Measurements of ultrasound images}

For longitudinal axis images, Achilles tendon insertion was divided into two distinct parts; insertion length and bone-to-insertion length. We defined the insertion length as the distance between proximal and distal attachment of the Achilles tendon insertion, while bone-to-insertion length was the distance between the superior edge of calcaneal bone to the proximal tendon attachment (figure 1). Similarly, for transverse images, we defined the width of the tendon as the distance between the medial to lateral tendon border and was measured by straight line (figure 2).

Two additional US measurements, tendon angle and cross-sectional area, were performed on healthy subjects (figure 3). The tendon angle was the angle between the upper part of the posterior surface of calcaneus and proximal attachment of tendon insertion. These measurements were not performed on the cadavers due to the embalmment and inability to standardize the effect of ankle position on these measurements.

Using measurement tools on OsiriX Lite version 8.0.2 software, two observers measured all cadaver images independently to assess validity and inter-tester measurement reliability. To minimize bias, both observers processed the US images blindly by performing measurements on the deidentified US images. For procedural reliability, the same person who collected the images of the healthy subjects, also performed the measurements but was blinded to information about subject and side for all images. The average of 4 measurements trials was used.

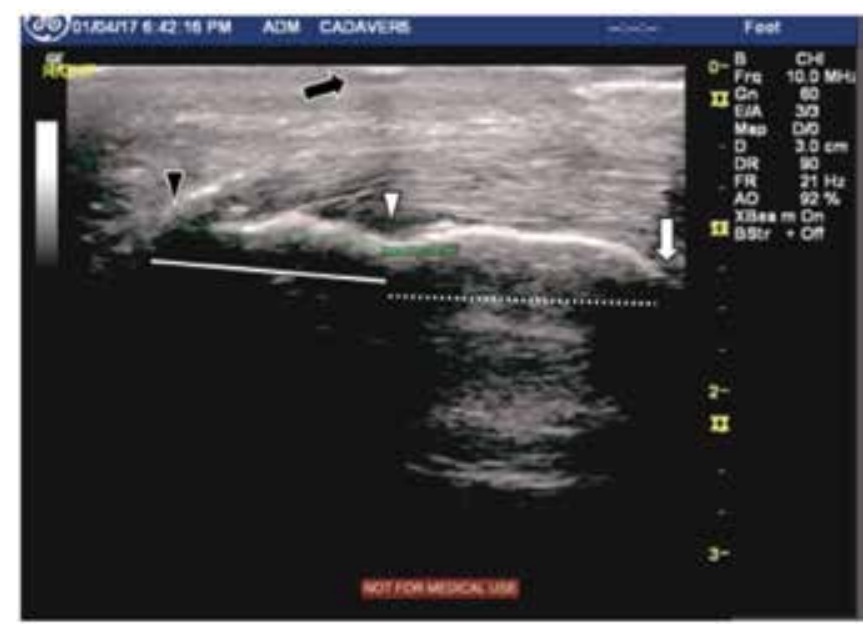

Figure 1. Long-axis US image of Achilles tendon insertion for a cadaver. The black arrow represented the tape shadow, the white arrow represented postero-superior part of the calcaneus, the white head arrow represented proximal attachment; the black head arrow represented distal attachment; the white solid line represented insertion length; and the white dots line represented bone-to-insertion length.

\section{Collecting anthropometric measurements}

After three weeks from obtaining the US images, dissections of the Achilles tendons were performed on the cadavers by first author to minimize the risk of bias by limiting the first author's ability to remembering the measurements values. The cadavers were placed in prone position with the ankle as close to a neutral position as possible. Skin was removed to expose the Achilles tendon; then, the fat pad and other connective tissues around the tendon were carefully detached. A digital caliper (Mitutoyo) was used for measuring the length and width of the insertional area in millimeter and repeated four times. We initially measured tendon width proximal to the calcaneal bone before detaching the tendon and measuring the length. Secondly, the tendon insertion was detached carefully from insertion site starting from the tendon edge distally removing the tendon fibers from insertion. The edge of the proximal attachment

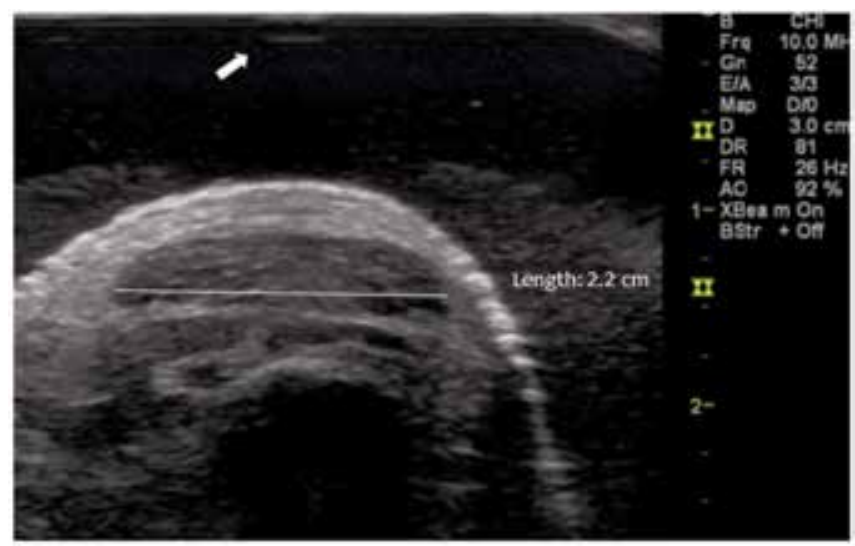

Figure 2. Short-axis US image of Achilles tendon at proximal to calcaneal bone; the white line represented the width and dash line cross-sectional area. The white arrow represented the tape shadow.

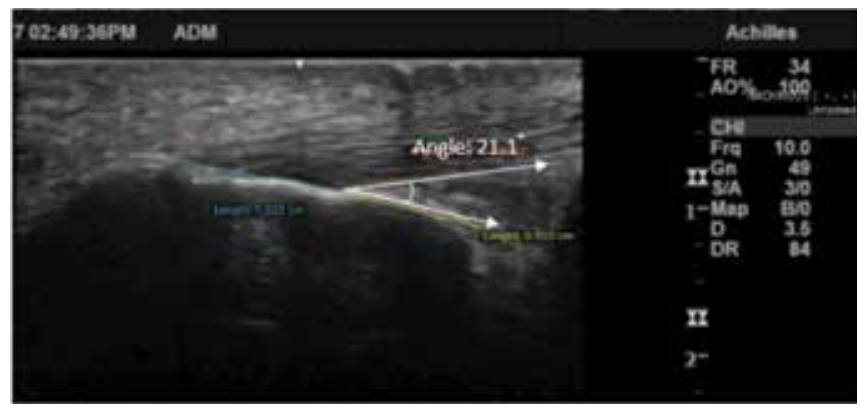

Figure 3. Long axis US image of Achilles tendon insertion for a healthy individual. The white two arrows represented the angle of tendon insertion. 
became detectable on the calcaneal bone and when the tendon was reflected (figure 4). The tendon foot print on the calcaneal bone was used only to measure the bone-to-insertion length by using the caliper to measure between the calcaneal edge and the proximal attachment. For insertion length, the tendon was reflected and flattened while taken the measurements. Insertion length was measured from the detectable edge of proximal attachment to distal border of tendon insertion (figure 4). Finally, four trials of each measurement were averaged and used for analysis.

\section{STATISTICAL ANALYSIS}

All statistical analyses were performed using SPSS statistical package version 24 (SPSS Inc, Chicago, IL). A paired t-test was used for testing the differences between the US imaging and cadaveric measurements. ICC estimates and their $95 \%$ confidence intervals were calculated based on a mean-rating $(\mathrm{k}=4)$, model 3 for procedural reliability, and model 2 for measurement reliability. For reliability, ICC values were interpreted as follows; value greater than 0.9 represented excellent reliability, values between 0.75 and 0.9 represented good reli- ability, values between 0.5 and 0.75 indicated moderate reliability, and value less than 0.5 was poor reliability (21).

Test-retest reliability and standard error of measurement (SEM) was calculated according to the following equation: $S E M=S D \sqrt{ }(1-r)$ where $S D$ is the standard deviation of the baseline measurement and $\mathrm{r}$ is the index of reliability (22). We also calculated the smallest detectable change $\left(\mathrm{SDC}_{95 \%}\right)$ for an individual, $\mathrm{SDC}_{95 \%}=2.77 \mathrm{x} \mathrm{SEM}$, and for the group level, $\mathrm{SDC}_{95 \%}=2.77 \times \mathrm{SEM} /$. For all inferential testing the level of significance was set at $\mathrm{p}<0.05$.

\section{RESULTS}

\section{Validity}

\section{Agreement between US images and cadaveric measurements}

There was good agreement between US and cadaver measurements for bone-to-insertion length $\left(\mathrm{ICC}_{2,4}=0.856\right.$ ) and tendon width before calcaneal bone ( $\operatorname{ICC}_{2,4}=0.840$ ), and poor agreement for insertion length $\left(\mathrm{ICC}_{2,4}=0.413\right)$ (table

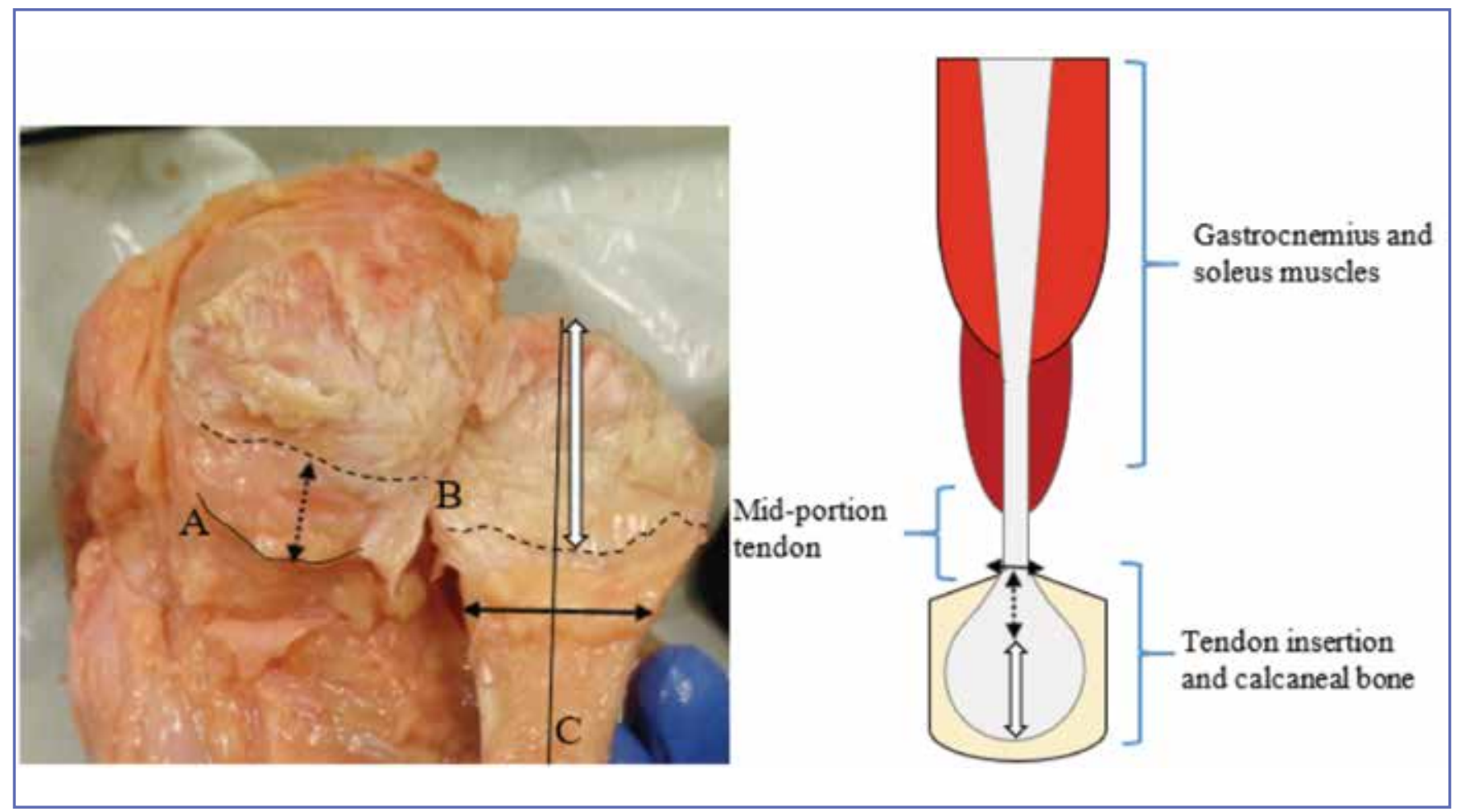

Figure 4. A, B and C lines represent calcaneal edge, proximal attachment cutting, and centerline, black arrow represented tendon width at calcaneal bone edge, black dots arrow represented bone-to-insertion length, white arrow represented insertion length. 
I). There were no significant differences between insertion length on US images and cadaveric measurements $(\mathrm{p}>0.05)$; however, there were significantly lower mean differences of cadaveric measurements for bone-to-insertion and tendon width before calcaneal bone $(\mathrm{p}<0.05)$ (table I).

\section{Reliability}

Inter-tester reliability of taking the measurements

We found that the agreement between observer 1 and 2 was good to excellent for insertion length $\mathrm{ICC}_{2,4}=0.784$ with $95 \%$ confident interval $=0.404-0.924$, bone-to-insertion length $\mathrm{ICC}_{2.4}=0.935$ with $95 \%$ confident interval $=0.820$ - 0.977, and tendon width before calcaneal bone $\mathrm{ICC}_{2,4}=$ 0.952 with $95 \%$ confident interval $=0.627-0.987$.

\section{Procedural Reliability - Test-retest reliability}

For the 6 healthies, (12 tendons, mean and standard deviation of age 25 (5.6), height $167 \mathrm{~cm} \mathrm{(6.7)} \mathrm{and} \mathrm{weight} 71.89$ $\mathrm{kg}(17.75))$. There were no significant differences in all measurements on US images between baseline and after 30 min ( $p>0.05)$. All measurements had excellent ICCs (0.9180.977) (table II). The standard error of measurement and smallest detectable change at the individual and the group level are listed in (table II).

\section{DISCUSSION}

This study was conducted to establish the reliability and the validity of US imaging for quantifying the Achilles tendon insertion area (insertion length, bone-to-insertion length, tendon width before calcaneal bone level, tendon angle and tendon cross-sectional area). The main findings suggest that using US imaging for measuring the Achilles tendon insertion length, bone-to-insertion, tendon angle, and tendon width and cross-sectional area at the level of before calcaneal bone are valid and reliable. While the intra-tester and procedural reliability was excellent for all measurements, the inter-tester reliability of measurements was good for insertional length, and excellent for other measurements. The validity of insertional length was found to be poor, but good for bone-to-insertion length and width before insertion.

\section{VALIDITY}

In this study, we evaluated the validity by using cadavers as our gold standard. Prior studies have used the cadavers agreement method for Achilles and patellar tendons length measurements as references and reported high level of agreement $(16,23)$. Our results suggest that two measurements; bone-to-insertion length and width before insertion, have good validity. While one measurement (insertional

Table I. Results of validity.

\begin{tabular}{|c|c|c|c|c|c|}
\hline Measurements location & $\begin{array}{l}\text { US observer } 1 \\
\text { Mean (SD) } \\
\mathrm{n}=16\end{array}$ & $\begin{array}{l}\text { Cadaveric } \\
\text { Mean (SD) } \\
\mathrm{n}=16\end{array}$ & ICC $_{2,4}$ & $(95 \% \mathrm{CI})$ & $\begin{array}{l}\text { Comparison } \\
\text { P value }\end{array}$ \\
\hline Insertion length $(\mathrm{mm})$ & $15.56(1.77)$ & $16.27(1.51)^{*}$ & .413 & $(-.609, .797)$ & 0.200 \\
\hline Bone-to-Insertion length (mm) & $11.97(2.34)$ & $11.18(2.04)$ & .856 & $(.561, .951)$ & 0.045 \\
\hline Before calcaneal bone width (mm) & $20.59(2.6)$ & $18.99(2.78)$ & .840 & $(.100, .956)$ & 0.001 \\
\hline
\end{tabular}
$* \mathrm{n}=15$

Table II. Results of test-retest reliability.

\begin{tabular}{|c|c|c|c|c|c|c|}
\hline Measurements location & $\begin{array}{l}\text { Test baseline } \\
\text { Mean (SD) } \\
\mathrm{n}=12\end{array}$ & $\begin{array}{l}<30 \text { min retest } \\
\text { Mean (SD) } \\
\mathrm{n}=12\end{array}$ & SEM & $\mathrm{ICC}_{3,4}(95 \% \mathrm{CI})$ & $\begin{array}{l}\text { SDC }_{95 \%} \\
\text { Individual }\end{array}$ & $\begin{array}{l}\mathrm{SDC}_{95 \%} \\
\text { group }\end{array}$ \\
\hline Insertion length (mm) & $12.95(3.49)$ & $13.39(3.33)$ & 0.529 & $.977(.92, .993)$ & 1.46 & 0.42 \\
\hline Bone-to-Insertion length (mm) & $9.34(2.21)$ & $9.06(2.40)$ & 0.442 & $.960(.866, .988)$ & 1.22 & 0.35 \\
\hline $\begin{array}{l}\text { Before calcaneal bone width } \\
(\mathrm{mm})\end{array}$ & $17.75(2.18)$ & $17.42(2.21)$ & 0.508 & $.946(.822, .984)$ & 1.41 & 0.41 \\
\hline $\begin{array}{l}\text { Tendon insertion angle } \\
\text { (degree) }\end{array}$ & $15.33(7.46)$ & $14.62(6.58)$ & 1.930 & $.933(.771, .98)$ & 5.35 & 1.54 \\
\hline
\end{tabular}


length) had poor validity, this might be due to some difficulties related to dissecting tendons from the cadavers. One explanation for poor value is the presence of calcification at distal insertion in some cadavers. Although we were able to measure Achilles tendon insertion with bony deformity distally on US images, it was difficult to perform measurements on some cadavers when the calcified tendon was hard to dissect. For this reason, one tendon was destroyed and had to be excluded from the statistical analysis. Also, it is a possible to include part of the connective tissue when dissecting the tendon from its insertion at distal end. In addition, a difficulty with using embalmed cadavers was that the stiffness of the ankle joint resulted in inability to standardize the ankle angle.

In this study the ankle position of cadaveric specimens varied from 15-65 degrees of plantar flexion. Our study showed that tendon width and bone-to-insertion length of actual measurements were consistently smaller than US measurements. This might be due to a dehydration effect of tissue as there was about six weeks between obtaining the images and performing the direct measurement. The effect of dehydration has previously been reported with alteration of biomechanical properties of cadaver tendon before and after two hours of exposure to room condition (23). It is possible these changes affected the anthropometric measurements and led to poor agreement with US measurements.

Two additional measurements cross-sectional area and angle were evaluated for inter-tester reliability but not for validity. The nature of these two measurements make having such measurement tool to measure them on cadaver is not feasible. However, simple and advanced geometry measurements (e.g. angle and area) can be easily done through advanced imaging software (OsiriX) on US images.

\section{Measurement Reliability - inter-tester reliability}

Two observers (NA, KGS) performed the measurements on the ultrasound images in a random order and autonomously to minimize bias. Although the first observer was the same person who did the cadaver measurement and obtained the images, the US measurements was performed blindly and after several weeks from measuring the actual insertion tendon area. The results above suggest that there is good to excellent agreement between observers, which supports the inter-tester reliability.

\section{Procedural Reliability - test-retest reliability}

Our study is the first that measured Achilles tendon cross-sectional area and width at insertion using bony landmark as a reference. Prior studies measured cross-sectional area and coronal diameter of the Achilles tendon at the level of medial malleolus or different distances from the calcaneal insertion $(25,26)$. The reproducibility of tendon CSA and tendon width was reported in previous studies but in different statistical method such as inter class correlation and coefficient of variance $(\mathrm{CV})$; however, the reported values as follow: tendon CSA as ICC $=.92(27)$, tendon CSA with $7 \% \mathrm{CV}$ and tendon width with $4 \% \mathrm{CV}$ (28); and tendon CSA with ICC $=0.81$ (25). Taking two measurements (width and area) at consistent location may help to explain the tendon formation at insertion level. As noted in prior studies tendon width and cross-section area become larger (oval shape) distally than at mid-portion proximally (26). Further research is needed to evaluate the measurements in different joint angles.

Knowing the true difference of each measurement is useful to distinguish error from true change; thus, the smallest detectable change values of each measurement was reported (table II). Very few studies reported SDC for Achilles tendon CSA. Our $\mathrm{SDC}_{95 \%}$ group of CSA was $4.74 \mathrm{~mm}^{2}$ comparing with $1 \mathrm{~mm}^{2}$ for CSA of free tendon (29), while other study reported $15 \mathrm{~mm}^{2}$ when CSA obtained at the level of medial malleolus (25). To our knowledge, no other studies have investigated the angle and insertion length.

Future studies should use these measurements to investigate the Achilles tendon insertion disorders development and prognosis with or without receiving surgical or non-surgical treatments around the Achilles tendon insertional structures.

\section{LIMITATIONS}

Our main study limitation was that some measurements were performed on healthy individuals. The purpose of that was to evaluate the stability of measurements on healthy people before testing it on patients with Achilles tendon insertion disorders. Also, because the cadavers were part of the anatomy class and the course instructor must follow the syllabus for educational purposes, we were not allowed to dissect the cadavers immediately after obtaining the US images; therefore, the considerable time gap between obtaining US image and dissecting Achilles tendon was unavoidable. Our dissection method may not be the ideal approach to get the real measurements precisely, but we followed a consistent technique for the most measurements to avoid any error throughout collecting the actual measurement. MRI is considered the gold standard for measuring anatomical structures and with it would be possible to compare the angle and cross-sectional area that were not possible to have a tool to measure it directly from the cadavers. It may be the insertional measurements change with changing the ankle joint ankle. More research is needed to establish the rela- 
tionship of changing of ankle joint angle with the insertional Achille tendon length measurements.

\section{CLINICAL IMPLICATION}

US imaging was found to be reliable and valid to determine Achilles tendon footprint length and tendon width. The use of this US imaging technique to determine Achilles tendon insertional size may be beneficial for evaluating the effect of structure on disease development and progression.

\section{REFERENCES}

1. Maffulli N, Sharma P, Luscombe KL. Achilles tendinopathy: Aetiology and management. J R Soc Med 2004;97:472-6. https://doi.org/10.1258/jrsm.97.10.472.

2. van Dijk CN, van Sterkenburg MN, Wiegerinck JI, Karlsson J, Maffulli N. Terminology for Achilles tendon related disorders. Knee Surgery, Sport Traumatol Artbrosc 2011;19:835-41. https://doi.org/10.1007/s00167-010-1374-z.

3. Kvist M. ACHILLES TENDON INJURIES IN ATHLETES 1991:188-201.

4. Malliaras P, Barton CJ, Reeves ND, Langberg H. Achilles and patellar tendinopathy loading programmes: A systematic review comparing clinical outcomes and identifying potential mechanisms for effectiveness. Sport Med 2013;43:267-86. https://doi.org/10.1007/s40279-013-0019-z.

5. Nørregaard J, Larsen CC, Bieler T, Langberg H. Eccentric exercise in treatment of Achilles tendinopathy. Scand J Med Sci Sport 2007;17:133-8. https://doi.org/10.1111/j.16000838.2006.00545.x.

6. Bleakney RR, White LM. Imaging of the Achilles Tendon. Foot Ankle Clin 2005;10:239-54. https://doi.org/10.1016/j. fcl.2005.01.006.

7. Kader D, Saxena a, Movin T, Maffulli N. Achilles tendinopathy: some aspects of basic science and clinical management. Br J Sports Med 2002;36:239-49. https://doi.org/10.1136/ bjsm.36.4.239.

8. Paavola M, Kannus P, Järvinen TAHT, Khan K, Józsa L, Järvinen M. Achilles tendinopathy. J Bone Joint Surg Am 2002;84A:2062-76. https://doi.org/10.1054/math.2002.0458.

9. Bernstein DN, Anderson MR, Baumhauer JF, Oh I, Flemister AS, Ketz JP, et al. A Comparative Analysis of Clinical Outcomes in Noninsertional Versus Insertional Tendinopathy Using PROMIS. Foot Ankle Spec 2018;XX:193864001880666. https://doi.org/10.1177/1938640018806662.

10. Richards PJ, Braid JC, Carmont MR, Maffulli N. Achilles tendon ossification: Pathology, imaging and aetiology. Disabil Rehabil 2008;30:1651-65. https://doi. org/10.1080/09638280701785866.

11. O’Brien EJO, Frank CB, Shrive NG, Hallgrímsson B, Hart DA. Heterotopic mineralization (ossification or calcification) in tendinopathy or following surgical tendon trauma. Int J Exp Pathol 2012;93:319-31. https://doi.org/10.1111/j.13652613.2012.00829.x.

\section{ACKNOWLEDGMENTS}

Research was supported by the Eunice Kennedy Shriver National Institute Of Child Health \& Human Development of the National Institutes of Health under Award Number K12HD073945 (MLK).

\section{CONFLICT OF INTERESTS}

The authors declare that they have no conflict of interests

12. Kang S, Thordarson DB, Charlton TP. Insertional Achilles Tendinitis and Haglund's Deformity. Foot Ankle Int 2012;33:487-91. https://doi.org/10.3113/FAI.2012.0487.

13. Mani-Babu S, Morrissey D, Waugh C, Screen H, Barton C. The effectiveness of extracorporeal shock wave therapy in lower limb tendinopathy: A systematic review. Am J Sports Med 2015;43:752-61. https://doi.org/10.1177/0363546514531911.

14. Özçakar L, Kara M, Chang KV, Çarl AB, Akkaya N, Tok F, et al. Nineteen reasons why physiatrists should do musculoskeletal ultrasound. Am J Phys Med Rehabil 2015; 94:e45-9. https:// doi.org/10.1097/PHM.0000000000000223.

15. Forney MC, Delzell PB. Musculoskeletal ultrasonography basics. Cleve Clin J Med 2018;85:283-300. https://doi. org/10.3949/ccjm.85a.17014.

16. Silbernagel KG, Shelley K, Powell S, Varrecchia S. Extended field of view ultrasound imaging to evaluate achilles tendon length and thickness: A reliability and validity study. Muscles Ligaments Tendons J 2016;6:104-10. https://doi.org/10.11138/ mltj/2016.6.1.104.

17. Cheng Y, Zhang J, Cai Y. Utility of Ultrasonography in Assessing the Effectiveness of Extracorporeal Shock Wave Therapy in Insertional Achilles Tendinopathy. Biomed Res Int 2016;2016:10-4. https://doi.org/10.1155/2016/2580969.

18. Chimenti RL, Chimenti PC, Buckley MR, Houck JR, Flemister AS. Utility of Ultrasound for Imaging Osteophytes in Patients with Insertional Achilles Tendinopathy. Arch Phys Med Rebabil 2016;97:1206-9. https://doi.org/10.1016/j.apmr.2015.12.009.

19. Del Baño-Aledo ME, Martínez-Payá JJ, Ríos-Díaz J, MejíasSuárez S, Serrano-Carmona S, de Groot-Ferrando A. Ultrasound measures of tendon thickness: Intra-rater, inter-rater and inter-machine reliability. Muscles Ligaments Tendons J 2017;7:192-9. https://doi.org/10.11138/mltj/2017.7.1.192.

20. Padulo J, Oliva F, Frizziero A, Maffulli N. Muscles, Ligaments and Tendons Journal - Basic principles and recommendations in clinical and field Science Research: 2018 update. MLTJ 2018; 8(3): $305-307$.

21. Koo TK, Li MY. A Guideline of Selecting and Reporting Intraclass Correlation Coefficients for Reliability Research. J Chiropr Med 2016;15:155-63. https://doi.org/10.1016/j. jcm.2016.02.012.

22. Denegar CR, Ball DW. Assessing reliability and precision of measurement: An introduction to intraclass correlation and standard error of measurement. J Sport Rehab 1993;2:35-42. 
23. Gellhorn AC, Morgenroth DC, Goldstein B. A Novel Sono graphic Method of Measuring Patellar Tendon Length. Ultra sound Med Biol 2012;38:719-26. https://doi.org/10.1016/j. ultrasmedbio.2012.01.020.

24. Verstraete MA, Van Der Straeten C, De Lepeleere B, Opsomer GJ, Van Hoof T, Victor J. Impact of drying and thiel embalming on mechanical properties of achilles tendons. Clin Anat 2015;28:994-1001. https://doi.org/10.1002/ca.22624.

25. Ying M, Yeung E, Li B, Li W, Lui M, Tsoi C-W. Sonographic evaluation of the size of achilles tendon: the effect of exercise and dominance of the ankle. Ultrasound Med Biol 2003;29:63742. https://doi.org/10.1016/S0301-5629(03)00008-5.

26. Martin KD, Wake J, Dawson L, Van Buren JP. Cross-sectional Area of the Achilles Tendon in a Cohort of Elite Military Warriors Using Standard Ultrasound Techniques. Mil Med 2018;00:1-4. https://doi.org/10.1093/milmed/usy019.
27. Dudley-Javoroski S, McMullen T, Borgwardt MR, Peranich LM, Shields RK. Reliability and responsiveness of musculoskeletal ultrasound in subjects with and without spinal cord injury. Ultrasound Med Biol 2010;36:1594-607. https://doi. org/10.1016/j.ultrasmedbio.2010.07.019.

28. Brushøj C, Henriksen BM, Albrecht-Beste E, Hölmich P, Larsen K, Bachmann Nielsen M. Reproducibility of ultra sound and magnetic resonance imaging measurements of tendon size. Acta Radiol 2006;47:954-9. https://doi. org/10.1080/02841850600854936.

29. Zellers JA, Cortes DH, Pohlig RT, Silbernagel KG. Tendon morphology and mechanical properties assessed by ultra sound show change early in recovery and potential prognos-tic ability for 6 month outcomes. Knee Surg Sport Traumatol Arthrosc 2018:in review. https://doi.org/10.1007/ s00167-018-5277-8. 